
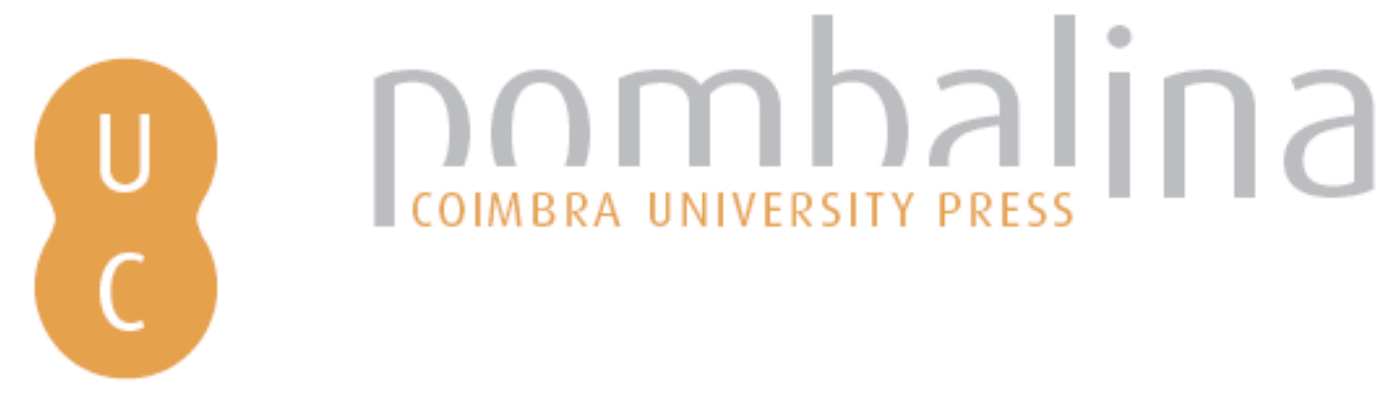

\title{
Algumas técnicas em ambiente SIG úteis à obtenção de áreas de serviço de conjuntos de pontos
}

\author{
Autor(es): $\quad$ Tralhão, Lino; Rodrigues, João Coutinho; Sousa, Nuno \\ Publicado por: Imprensa da Universidade de Coimbra \\ URL \\ persistente: URI:http://hdl.handle.net/10316.2/37101 \\ DOI: $\quad$ DOI:http://dx.doi.org/10.14195/978-989-26-0983-6_48 \\ Accessed : $\quad$ 26-Apr-2023 11:22:54
}

A navegação consulta e descarregamento dos títulos inseridos nas Bibliotecas Digitais UC Digitalis, UC Pombalina e UC Impactum, pressupõem a aceitação plena e sem reservas dos Termos e Condições de Uso destas Bibliotecas Digitais, disponíveis em https://digitalis.uc.pt/pt-pt/termos.

Conforme exposto nos referidos Termos e Condições de Uso, o descarregamento de títulos de acesso restrito requer uma licença válida de autorização devendo o utilizador aceder ao(s) documento(s) a partir de um endereço de IP da instituição detentora da supramencionada licença.

Ao utilizador é apenas permitido o descarregamento para uso pessoal, pelo que o emprego do(s) título(s) descarregado(s) para outro fim, designadamente comercial, carece de autorização do respetivo autor ou editor da obra.

Na medida em que todas as obras da UC Digitalis se encontram protegidas pelo Código do Direito de Autor e Direitos Conexos e demais legislação aplicável, toda a cópia, parcial ou total, deste documento, nos casos em que é legalmente admitida, deverá conter ou fazer-se acompanhar por este aviso.

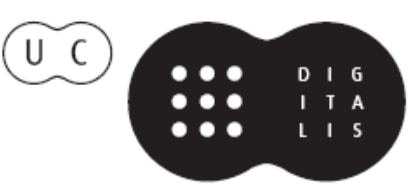




\section{$\forall$ \\ TAS DAS I JORNADAS LUSÓFONAS DE CIÊNCIAS E TECNOLOGIAS DE INFORMAÇÃO GEOGRÁFICA}

Editores

José Gomes dos Santos

Cidália Fonte

Rui Ferreira de Figueiredo

Alberto Cardoso

Gil Gonçalves

José Paulo Almeida

Sara Baptista 


\title{
ARTIGO 48
}

\section{Algumas técnicas em ambiente Sig Úteis à OBTENÇÃo de ÁREAS de SERViço de CONJUNTOS DE PONTOS}

\author{
TRALHAO, Lino ${ }^{1}$; COUTINHO-RODRIGUES, João ${ }^{2}$; SOUSA, Nuno 3
}

\begin{abstract}
${ }^{1}$ Instituto de Engenharia de Sistemas e Computadores (INESC-Coimbra); Rua Antero Quental 199, 3000033 Coimbra, Portugal; Tel: +351 239 851040/9; Fax: +351 239 824692; email: linotralhao@netcabo.pt 2 Departamento de Engenharia Civil da Faculdade de Ciências e Tecnologia da Universidade de Coimbra Instituto de Engenharia de Sistemas e Computadores (INESC-Coimbra); Departamento de Engenharia Civil - FCTUC; Rua Luís Reis Santos - Pólo II, 3030-788 Coimbra, Portugal; Tel: +351 239 797145; Fax: +351239 797123; email: coutinho@dec.uc.pt

3 Departamento de Ciências e Tecnologia da Universidade Aberta; Instituto de Engenharia de Sistemas e Computadores (INESC-Coimbra); Delegação de Coimbra da Universidade Aberta; Rua Alexandre Herculano, n. ${ }^{\circ}$ 52, 3000-019 Coimbra, Portugal; Tel: +351300 001590; Fax: +351300 001599; email: nsousa@uab.pt
\end{abstract}

\section{RESUMO}

Em estudos de acessibilidade, e não só, são muito úteis um tipo de estruturas que se podem obter a partir de uma rede, eventualmente multi-modal e parametrizável: as chamadas "áreas de serviço", as quais são constituídas por polígonos, cada qual correspondente a uma zona situada entre um certo intervalo de custo, relativamente a uma certa "feature" (ponto, multiponto, etc.). Pretende-se neste estudo obter, a partir de áreas de serviço relativas a um universo de features, áreas de serviço relativas a subconjuntos dessas features. Estas técnicas envolvem manipulações relativamente complexas de polígonos e podem ser generalizadas para conjuntos de conjuntos e assim sucessivamente. Convém notar que nem sempre se dispõe da rede, pondendo dispôr-se das referidas estruturas; eventualmente, no caso de áreas de serviço, sob a forma de imagens (raster) a serem convertidas para formato vectorial. 
Atas das I Jornadas Lusófonas de Ciências e Tecnologias de Informação Geográfica, Sessão 11, Artigo 48 Algumas técnicas SIG úteis à obtenção de áreas de serviço de conjuntos de pontos

Lino Tralhão, João Coutinho-Rodrigues \& Nuno Sousa

\title{
PALAVRAS-CHAVE
}

Acessibilidade a conjuntos de pontos, Sistemas de informação geográfica, ArcGIS, Áreas de serviço.

\section{SOME GIS ENVIRONMENT TECHNIQUES USEFUL FOR OBTAINING SERVICE AREAS OF SETS OF POINTS}

\begin{abstract}
In studies of accessibility, but not only, it is useful to consider, among others, a type of structures that can be obtained from a network, eventually multimodal and parameterizable: the so-called "service areas", which are made out of polygons, each corresponding to a zone between a certain impedance interval, with respect to a certain feature (point, multipoint, etc.). This study aims at obtaining, form service areas relative to a universe of features, service areas relative to subsets of those features. These techniques deal with relatively complex polygon manipulations and can be generalized for sets of sets, and so forth. It is worth noting that the network may not always be available, although the aforementioned structures usually are; eventually, for service areas, under the form of (raster) images, to be converted into vector format.
\end{abstract}

\section{KEYWORDS}

Accessibility to sets of points, Geographical information systems, ArcGIS, Service areas.

\section{INTRODUÇÃO}

O conceito de acessibilidade, aqui visto como respeitante aos benefícios para a população de aceder a certos locais, também ditos oportunidades, usando um determinado sistema de transporte (DE MONTIS \& REGIANNI, 2012), tem sido frequentemente usado em estudos científicos e joga um papel reconhecidamente importante na definição das políticas públicas (GEURS \& VAN WEE, 2004; BOCAREJO et al., 2012). Tratando-se de um conceito muito abrangente, este conceito é naturalmente dado a diferentes abordagens, o que de facto se verifica na literatura. Do ponto de vista clássico, a acessibilidade foi definida como "o potencial de oportunidades de interação" (HANSEN, 1959), "a facilidade com que qualquer atividade localizada pode ser alcançada a partir de outras localizações, usando um determinado sistema de transporte" (DALVI \& MARTIN, 1976) ou 
"os benefícios oferecidos por um sistema de ordenamento de território/ transportes" (BEN- AKIVA \& LERMAN, 1979). Outras abordagens foram usadas por diferentes autores (e.g. GEURS \& VAN ECK, 2001; LITMAN, 2003; HALDEN et al., 2005). Recentemente tem havido um recrudescer do interesse em análises de acessibilidade e estudos afins (DE MONTIS \& REGIANNI, 2012), continuando tais análises a ser centrais na pesquisa sobre assuntos regionais e urbanos (PÁEZ et al., 2012). Como reconhecido por IACONO et al. (2010), o melhoramento da acessibilidade, vista como a facilidade em alcançar os destinos procurados, dada uma gama de oportunidades disponíveis e impedâncias ("custos", e.g. tempo, comprimento do caminho mais curto, etc.) associadas aos recursos usados na deslocação entre origens e destinos, emergiu recentemente como um dos objetivos centrais aos decisores ligados ao planeamento urbano.

Neste cenário, os Sistemas de informação geográfica (SIG) (GEERTMAN \& VAN ECK, 1995; LONGLEY et al., 2011; HEYWOOD et al., 2011; MAVOA et al., 2012) aparecem como uma ferramenta natural para executar a tarefa. O SIG permite guardar e representar informação georreferenciada de natureza espacial. Permite também analisar, manipular e operar sobre essa informação. Os ambientes SIG dispõem de "ferramentas" ("tools") para algumas destas operações, mas é frequentemente necessário estender-se as funcionalidades do SIG desenvolvendo ferramentas analíticas adicionais, destinadas a lidar com problemas específicos. A tecnologia SIG tornou-se assim essencial a estudos de acessibilidade, sendo frequentemente útil no planeamento e gestão da cidade e do território.

É neste contexto que se pretende apresentar algumas técnicas úteis ao estudo da acessibilidade a equipamentos cuja representação em termos de múltiplos pontos pode ser de conveniência. P.ex. se um equipamento tiver dimensões suficientemente grandes, a sua representação por um só ponto pode ser grosseira quando a separação espacial (medida como distância, tempo de viagem ou qualquer outra variável de impedância espacial - ver p.ex. POOLER, 1995) ao ponto representativo mais próximo pode ser considerada ao invés.

Na secção 2 deste trabalho (motivação), apresenta-se o tema do trabalho, na secção 3, duas técnicas para obtenção de áreas de serviço (SA) de conjuntos de pontos representativos, e também uma técnica 
para a obtenção de um indicador de acessibilidade. Como exemplo de aplicação destas técnicas, é apresentado, na secção 4, um caso de estudo envolvendo as paragens de 14 linhas de autocarro na cidade de Coimbra, Portugal. Na secção 5 são tecidas algumas considerações de conclusão sobre o trabalho.

\section{MOTIVAÇÃo}

Antes de mais convém notar que este trabalho foi realizado no contesto do ambiente ArcGIS Desktop 10.1. Aqui se considera a impedância a (ou de) um equipamento representado por um conjunto de pontos, como a menor das impedâncias a (ou de) esses pontos. Dado que se lida neste trabalho com áreas de serviço (ESRI, 2007), estas, quando relativas a um conjunto de pontos assentam na "menor impedância" acima referida.

É sabido que, no contexto da extensão Network Analyst (NA), pode obter-se áreas de serviço relativas a estruturas de tipo multiponto. No entanto, estas são obtidas relativamente a um dos elementos do multiponto. O NA permite, contudo, obter áreas de serviço relativas a um conjunto de pontos (aqui designados por conjuntos multiponto). Porém, se se tiver um universo de pontos e se quiser áreas de serviço relativas a uma classe de subconjuntos desse universo, essa obtenção terá de ser feita introduzindo, um a um, e correndo o NA para cada desses subconjuntos o que, tendo em conta o facto de em geral tais corridas serem demoradas, pode tornar o processo muito demorado e também bastante maçador (excepto usando um script ou o Model Builder). Além disso, usar esta funcionalidade requer ter-se uma rede onde a supra-citada extensão do ArcGIS irá operar e, mais ainda, tê-la programado com parâmetros adequados, nem sempre conhecidos.

Acontece que nem sempre se dispõe ou da rede ou da possibilidade de a usar convenientemente, p.ex. por não se dispor do próprio NA ou porque a programação/configuração do NA, por muito complexa ou envolvendo certos parâmetros, estará disponível noutro local/entidade. É pois conveniente solicitar a essa entidade áreas de serviço relativas aos vários pontos desse universo e, localmente, e por manipulação desses polígonos, obter SA para subconjuntos desse universo, considerando 
inclusivamente diferentes partições deste.

Mas mesmo dispondo-se da rede e do NA devidamente programado/ configurado, a obtenção de SA para conjuntos de pontos pode não ser possível, ou, pelo menos, ser muito complexa de obter. É o que acontece, por exemplo quando se trata de uma rede multimodal e pretende-se estudar como se encontram situadas uma ou mais zonas de uma cidade, considerando simultaneamente, SA agregadas relativamente a diferentes modos de transporte.

Mais ainda, pode de todo não existir rede, pelo que o NA nem sequer chega a ser útil. Neste caso os polígonos relativos a SA são obtidos por outras vias, eventualmente em formato raster (a serem convertidos em formato vetorial) ou mesmo já em formato vetorial. Muitos serão os exemplos que poderão ser fornecidos desde zonas de influência de bases navais ou aéreas, até SA demarcadas no terreno, tendo em conta as suas irregularidades. Seja, por exemplo, uma zona florestal com várias entradas possíveis, e, para cada entrada e para cada tipo de veículo, as zonas de penetração desses veículos, correspondentes a diferentes intervalos de tempo; pretende-se determinar qual ou quais as combinações (subconjuntos) de entradas a serem utilizadas num incêndio.

Um outro problema relacionado com áreas de serviço reside em que não raramente se trata de polígonos definidos por muitos vértices, frequentemente da ordem dos milhões. Assim, a sua obtenção usando NA pode originar "crashes" computacionais, em geral resultantes de problemas de memória. Uma forma de tornear esta dificuldade consiste em usar-se uma quadrícula ("mesh"). Por métodos que aqui se omitem (mas que o algoritmo $C$ sugere) e com precisão relacionada com o lado das células da mesh, podem obter-se áreas de serviço a partir de matrizes origem-destino (OD) em que as origens (destinos) são os pontos de, e os destinos (origens) os centróides das células da mesh. É sabido que as heurísticas usadas pelo NA na obtenção das impedâncias elementos das matrizes OD são relativamente mais "ligeiras" do que as relativas a áreas de serviço, logo menos propensas a problemas de memória. No entanto, o uso de matrizes OD no Network Analyst não permite o tipo de estudo que as áreas de serviço permitem, porque o que acontece é que cada elemento de uma matriz OD relativa a objectos do tipo multiponto 
contêm a impedância a um dos pontos da lista em que internamente o multiponto é representado, e não a impedância ao ponto "mais próximo" de cada multiponto.

\section{METODOLOGIA UTILIZADA}

Vai pois partir-se do princípio de que se dispõe de uma "pool" de áreas de serviço relativas a pontos de um certo conjunto,. Dessa pool pode, usando algoritmos que aqui se apresentam, obter-se áreas de serviço relativas a subconjuntos de, aqui designados por "conjuntos multiponto". Assim, pode obter-se conjuntos de áreas de serviço, para diferentes configurações desses subconjuntos, sem necessidade de recurso sistemático ao Network Analyst. De notar que essa pool pode ser obtida a partir de imagens (raster) e depois, por algum dos modos possíveis, convertida ao formato vectorial.

Antes de prosseguir para a apresentação das técnicas, convém esclarecer alguns conceitos. Neste trabalho o conceito de "feature class" (FC) (BUTLER, 2008) é usado no sentido de um conjunto de "features", todas elas com o mesmo tipo de geometria e no mesmo sistema de geo-referenciação. Este é um sentido um pouco mais lato do que o do ArcGIS (ESRI, 2014), incluindo conceitos como p.ex. shapefile, "feature layer", "feature set". Apesar de algumas ferramentas requererem um tipo específico de dados de entrada, no geral não é esse o caso, além de que há métodos adequados para a conversão entre tipos de dados. Por outro lado, cada FC tem associada uma tabela (no sentida da Teoria das Bases de dados - um SIG engloba como sua peça chave uma ou mais Base de Dados onde todos os seus objectos se encontram codificados) onde um conjunto de atributos ("fields" ou campos) pertinentes aos seus objectos (features) são especificados, bem como os valores que cada feature possui em cada atributo. Cada coluna da tabela corresponde a um atributo, cada linha corresponde a uma feature, encontando-se nessa linha os valores desse objecto nos vários atributos. Temos pois uma quarta característica de uma FC - os seus objectos estão associados a um conjunto comum de atributos. Ao conjunto de atributos de uma tabela chamamos "schema". Alguns desses atributos são criados pelo próprio ambiente, outros são da 
responsabilidade do utilizador o qual dispõe de ferramentas para criar ou eliminar atributos deste segundo tipo.

Outro conceito pertinente é o de junção (Join) que aqui se representa por $J_{m}^{t}$, onde os significados de $\mathrm{t}$ e $\mathrm{m}$ serão esclarecidos no seguimento. Existem fundamentalmente dois tipos de junções: as de atributo $(t=a)$ e as espaciais $(t=s)$.

As junções de atributo dizem respeito essencialmente às tabelas associadas às FC operandas e correspondem às operações, com o mesmo nome, da álgebra relacional da Teoria das Bases de Dados. Na junção $x_{1} J_{m}^{a} x_{2}$, em que $x_{1}$ e $x_{2}$ são FC (operandos) e $m$ um campo (ou tuplo de campos) chave (aqui designado por "chave estrangeira"), as features do resultado são, tal como acontece na junção espacial, as features de $x_{1}$ ( $1^{\circ}$ operando); porém a tabela tem por schema a união dos schemas dos operandos, vindo primeiro (colunas da esquerda) os atributos de $x_{1}$ e a seguir os de $x_{2}$. Tratando-se de assunto bem conhecido da Álgebra Relacional - Teoria das Bases de Dados relacionais - não será aqui mais desenvolvido. Porém convém referir que o ArcGis somente respeita o especificado na Álgebra Relacional para relações do tipo m:1. Para relações $\mathrm{m}: \mathrm{n}$, e para cada valor da chave estrangeira escolhe somente a $1^{\mathrm{a}}$ linha do $2^{\circ}$ operando que satisfaz a junção, não procedendo a qualquer replicação de linhas/features do $1^{\circ}$ operando. Assim, estas junções podem ser "desfeitas", ficando o $1^{\circ}$ operando com a sua tabela "separada" da do $2^{\circ}$. Como o ArcGis só permite um campo como chave estrangeira, para junções em que esta constitui um tuplo de campos deve criar-se um campo auxiliar que agregue os valores relativos ao tuplo. P.ex. numa junção (multi-atributo) $\mathrm{m}=(k e y G, \operatorname{key} Q M)$, cria- se em ambas as tabelas dos operandos um campo de tipo string, $k e y G \_k e y Q M$, preenchendo-o (Calculate Field) usando a expressão [keyG] \&"_"\& [keyQM] (caso se use VBA); este campo será a chave estrangeira da junção.

Na junção espacial ( $t=s$ ) (ferramenta Spatial Join) não estão em causa quaisquer valores de atributos mas relações de tipo geométrico entre as features dos operandos (relações tipo "within", “inside”, etc.). Por exemplo na junção $x_{1} J_{W I T H I N}^{S} x_{2}$, features de $x_{1}$ continuam a estar presentes no resultado; existe também o prolongamento da tabela de $x_{1}$ referido acima, só que o prolongamento da linha da tabela relativa a uma feature de 
$x_{1}$ acontece se essa feature estiver dentro de uma feature de $x_{2}$, sendo prolongada pelos valores nos atributos desta feature de $x_{2}$. Aqui pode haver replicação de features de $x_{1}$; é o que acontece quando a relação geométrica em causa é verdadeira para mais do que uma features de $x_{2}$ (e, além disso, é escolhida uma opção, “join_one_to_many”). O resultado é colocado numa $3^{\mathrm{a}}$ FC. Em anexo apresentam-se as ações executadas por ferramentas relevantes aos algoritmos apresentados.

Dada a sua complexidade, serão aqui explicitadas mais detalhadamente três ferramentas. A $1^{\text {a }}$ é a ferramenta Union, aqui representada pelo símbolo $\boldsymbol{U}$. Para mostrar a sua acção vamos recorrer à Fig 1. Tal como em outras figuras, as "features" aparecem desniveladas e em perspectiva somente para melhor explicitação. De facto todas encontram-se no mesmo plano. Usa-se a expressão "em perspectiva”, quando tal desnivelamento é dado.

A figura 1 apresenta, do lado esquerdo, duas FC de polígonos: a FC galaxies e a FC MasterDiss100. As features aparecem separadas em perspectiva. Dando transparência às features de modo a só se ver as respectivas fronteiras, temos, do lado esquerdo da figura 1 , o mesmo conjunto de features visto de cima. Aqui se pode ver que, dado situarem-se no mesmo plano, as features das duas FC se retalham reciprocamente de modo a termos um conjunto de "pedaços" (divisões). A ferramenta Union tem por resultado uma FC constituída por essas divisões. O sche$m a$ do resultado é essencialmente a união dos schemas dos operandos (até ao sistema 9); a partir do sistema 10 existe uma opção relativa ao schema, a opção que mais interessa aqui é "No FiD”, que corresponde à referida união de "schemas". Vamos supor que é criado, em cada das FC um campo que tem por função constituir uma chave candidata (um chave candidata é um campo cujos valores se distribuem sem ambiguidade pelas features de uma FC ou pelas linhas de uma tabela, podendo assim identificá-las univocamente). Chamemos a esse campo keyG e $G N r$, respectivamente na FC galaxies e na FC MasterDiss100 (vide figura 1). Cada divisão sobrepõe-se a uma ou mais features das FC operandas. Ora, no resultado vão aparecer os campos $k e y G$ e $G N r$.

Os valores atribuidos a esses campos indicam a feature de galaxies e a feature de MasterDiss100 a que a divisão se sobrepõe. Se uma divisão se 
sobrepuser a mais do que duas features do mesmo operando, então essa divisão na realidade corresponde a vários polígonos iguais e sobrepostos, ou seja, o respectivo polígono encontra-se replicado. Assim (vide figura 2), uma divisão que se sobreponha às features 1 e 2 de galaxies e às features 2 e 3 de MasterDiss100, corresponde no resultado a 4 polígonos iguais e sobrepostos: $(k e y G, G N r)=(1,2),(1,3),(2,2),(2,3)$. Se uma divisão se sobrepuser somente a features de um dos operandos, surgirá no resultado tantas vezes quantas essas features, mas com valor 0 (ou Null) na chave correspondente ao outro operando. P.ex. uma divisão que se sobreponha às features 1 e 3 de mas a nenhuma de MasterDiss100, terá duas réplicas: $(k e y G, G N r)=(1,0),(3,0)$. Isto significa que podemos encarar essas "sobreposições" como "proveniências", pelo que do resultado de uma Union se podem reconstruir os operandos através do uso da ferramenta Dissolve sobre a chave respeitante a esse operando. $\mathrm{Na}$ ferramenta Intersect, que aqui se representa pelo símbolo $\bigotimes$, tudo se passa similarmente à Union, mas com uma diferença: somente surgem (replicadas ou não) as divisões que se sobrepõem a features de ambos os operandos, portanto, nos tuplos acima não haverá zeros.
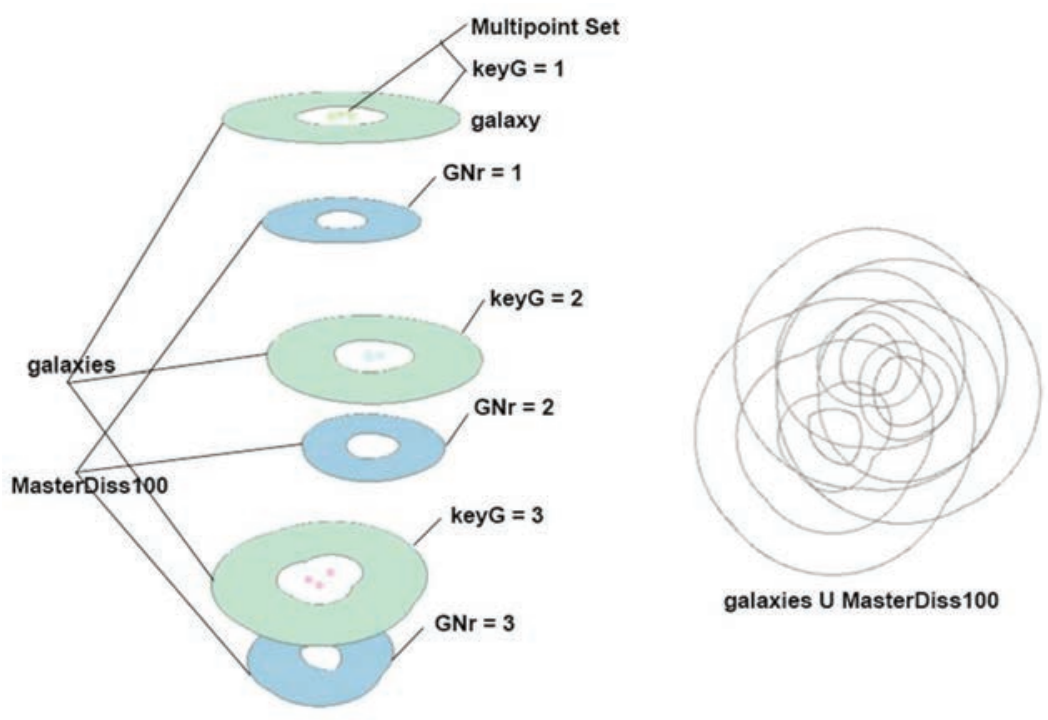

Figura 1 - Union 


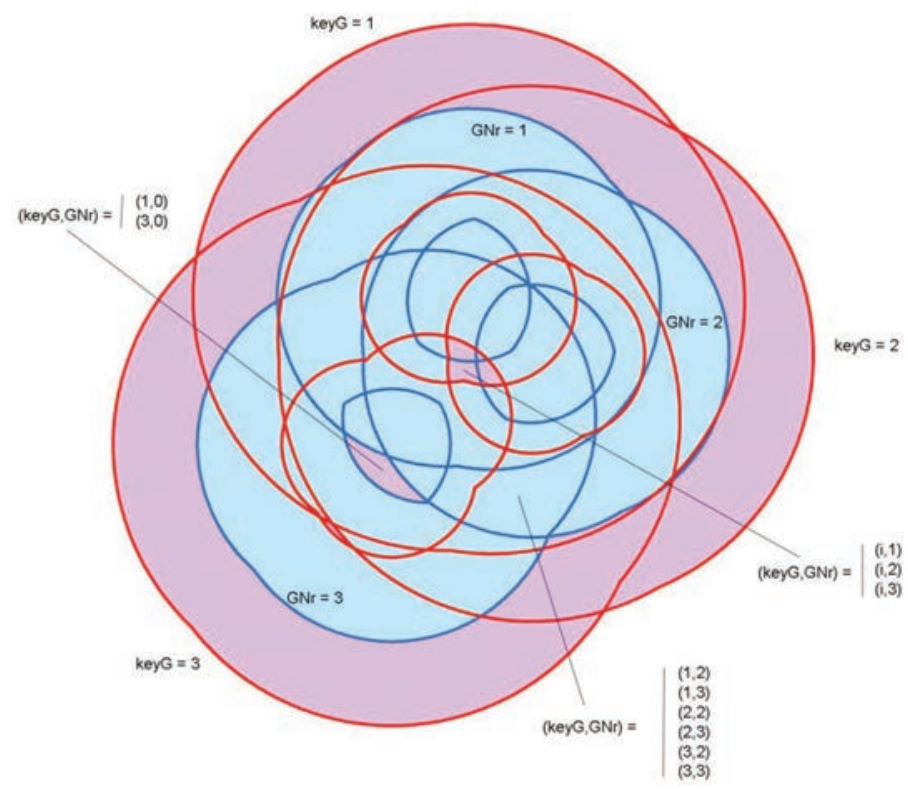

Figura 2 - Simmetrical Difference e Intersect

Na ferramenta Symmetrical Difference, que aqui se representa pelo símbolo $\Delta$, surgem as divisões da Union que não pertencem ao resultado da ferramenta Intersect, portanto as divisões que se sobrepõem a features de somente um dos operandos. Portanto para todo o par - (keyG, $G N r$ ) - um dos componentes é zero. Na figura 2, encontram-se representadas a intersecção e a diferença simétrica. De notar que as features da "intersecção" juntamente com as da "diferença simétrica" constituem as features da "união".

Os resultados das três ferramentas acima referidas podem apresentar features multipart, i.e. features contendo mais do que um elemento geométrico (p. ex. dois polígonos). Na aplicação dos algoritmos abaixo apresentados, tal não constitui problema de maior; porém pode usar-se a ferramenta Multipart to Singlepart para decompor essas features. É, porém, grave o caso de surgirem divisões com "geometria nula" (polígonos de área nula), ou então polígonos que se auto-intersectam (imagine-se, 
p.ex. uma ferradura que é "apertada" de modo à suas pontas se penetrarem mutuamente, mas mantendo os respectivos bordos). O ArcGis não lida bem com objetos destes tipos que considera como contendo erros de geometria. É pois aconselhável o uso da ferramenta Repair Geometry após qualquer daquelas operações a qual elimina os objetos de geometria nula e decompõe os que se auto-intersectam.

Uma outra operação que surge nos algoritmos é a atribuição, representada pelo símbolo $\leftarrow$. Esta operação pode corresponder a uma sequência operações se, porventura atribuir o resultado de uma operação (p.ex. $\cap$ ) a um dos seus operandos. O ArcGis não permite tal, salvo algumas exceções. Deverá primeiro colocar-se o resultado da operação (lado direito da atribuição) numa FC auxiliar e depois copiar esta para o destino final (lado esquerdo). Isto pode envolver ainda mais ações, mas um utilizador minimamente familiarizado com este ambiente saberá como proceder.

\section{1. Áreas de serviço a um subconjunto de featues - algoritmo A}

Vamos supor um conjunto $P$ de features, as quais, sem perda de generalização, consideramos como sendo pontos. Vamos também supor que temos um conjunto de sequências de $k \mathrm{SA}$, cada qual relativa a um ponto, e que, sem perda de generalização, se trata de áreas de serviço de tipo "ring", sendo que cada "ring" (ou "anel") corresponde a um intervalo de impedância (medido na rede, caso esta exista). Temos pois uma sequência $\left\{I_{1}, \ldots, I_{k}\right\}$ de intervalos de impedância adjacentes mas disjuntos. Assim, a cada ponto, $j$, corresponde uma sequência $\left\{\right.$ ring $^{j}{ }_{I 1}$, ..., $\operatorname{ring}_{I k} j_{\text {\} }}$ de áreas de serviço. A cada intervalo associamos um número identificador (chave), $\operatorname{key} Q M_{i}$.

Seja MasterSA uma FC onde se encontram todas as áreas de serviço correspondentes a todos os pontos de $P$. Portanto em MasterSA estará $\operatorname{Merge}\left(\operatorname{ring} j_{I 1}, \ldots, \operatorname{ring} j_{I k, \ldots}, \operatorname{ring} j_{I 1}, \ldots, \operatorname{ring} j_{I k}, \ldots\right)$, referindo a ferramenta Merge. Um dos campos da sua tabela associada será o campo keyQM. Terá também mais campos: um que identifica o ponto a que cada área de serviço (ring) corresponde; dois outros, $\operatorname{minImp}(\operatorname{maxIMP})$ que indicam o extremo inferior (superior) do intervalo de impedância correspondente a um ring; e eventualmente outros campos com demais informação. 
Consideremos agora subconjuntos (conjuntos multiponto) de $P$, disjuntos entre si, representados por $S_{k e y G}$. O índice keyG identifica (chave) o conjunto multiponto, e corresponde em MasterSA ao campo keyG que indica, para cada SA, qual o conjunto multiponto a que o ponto correspondente a essa SA pertence. Os conjuntos multiponto podem não ser exaustivos: podem corresponder somente a uma parte de $P$.

Na figura 1 aparecem 9 pontos agrupados em subconjuntos de três. Na figura 3 apresenta-se em perspectiva um desses conjuntos multiponto e áreas de serviço (tomadas como circulares) relativas aos seus pontos.

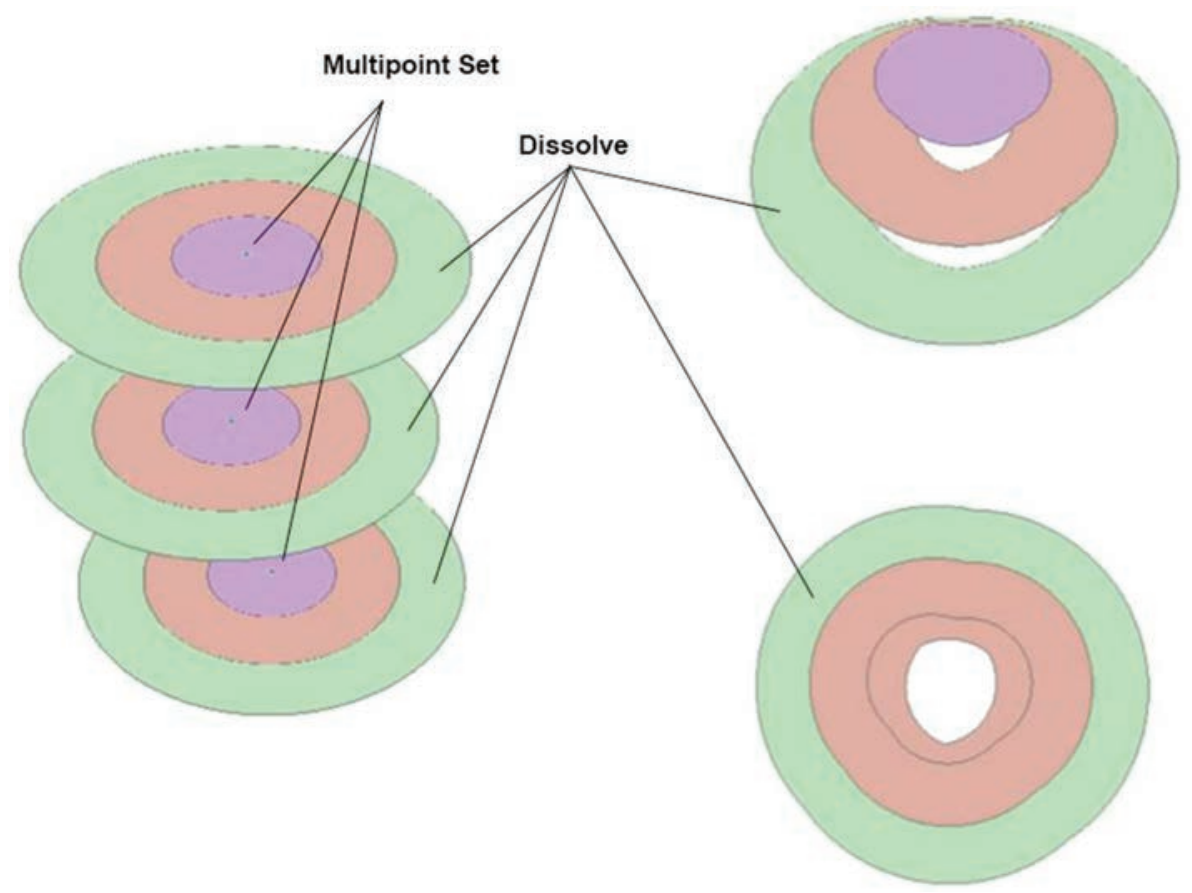

Figura 3 - SA de um subconjunto multiponto

Nesta figura aparece também o resultado da ferramenta Dissolve sobre keyQM, ou seja as uniões geométricas ("fusões") das SA referentes ao mesmo intervalo (representadas na mesma cor). Pode verificar-se que os anéis se sobrepõe. $\mathrm{Na} 3^{\mathrm{a}}$ parte da figura, agora sem desnivelamentos, mas com a SA referente ao intervalo inferior, transparente mas de modo a ver-se a sua fronteira, tal sobreposição está bem patente. Cada desses 
rings corresponde, no algoritmo $\mathrm{A}$, a ring ${ }_{I i}$ yey obtido no passo $\mathrm{A} 2$. a; no algoritmo B corresponde a um das features de MasterDiss.

Posto isto, o algoritmo A permite obter, a partir de MasterSA, as áreas de serviço correspondentes aos diferentes multipontos.

Para cada subconjunto multiponto $\left(S_{\text {keyG }}\right)$ :

A1. Determinar, a partir de MasterSA e usando a ferramenta Dissolve, a união geométrica, galaxy, de todas as SA com respeito a todos os pontos de keyG; (Nota: a FC galaxy é uma FC variável neste algoritmo.)

A2. Para $i=1$ a $k-1$ :

a. Determinar, a partir de MasterSA e usando a ferramenta Dissolve, a fusão, ring $g_{I_{i}}^{k e y G}$, das SA com respeito aos pontos de keyG correspondentes ao intervalo $I_{i}$;

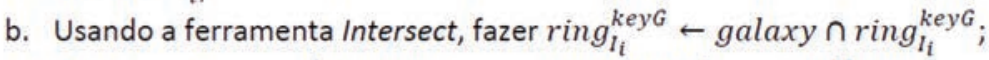

c. Com a ferramenta Symmetrical Difference, fazer galaxy $\leftarrow$ galaxy $\Delta \operatorname{ring}_{I_{i}}^{k e y G}$;

A3. ring $_{I_{k}}^{\text {keyG }} \leftarrow$ galaxy;

Para cada subconjunto multiponto $S_{\text {keyG }}$, as SA são $\left\{\right.$ ring $_{l_{1}}^{\text {keyG }}, \ldots$, ring $\left._{l_{k}}^{\text {keyG }}\right\}$ (ferramentas Merge ou Append)

Algoritmo A

Todas as SA obtidas, para todos os intervalos de impedância, e para todos os conjuntos multiponto, podem ser colocadas numa FC resultado, $s a$, no fim do ciclo exterior (usando a ferramenta Merge), ou por actualizações sucessivas de (ferramenta Append) - vide algoritmo B, passos B5 e B7.

Neste algoritmo, tal como nos seguintes, existem ou podem existir passos intermédios; ou seja, cada "instrução" pode corresponder a mais do que uma operação em ambiente SIG. Em geral trata-se da necessidade de lidar com resultados/passos intermédios, de "arranjos" dos esquemas "Schemas" (conjunto de campos - "field map") durante/após a execução de um passo, e também da já referida reparação de geometria.

$\mathrm{Na}$ figura 4 apresentam-se os passos A2 a, b e c, para $k e y G=1, i=2$. 


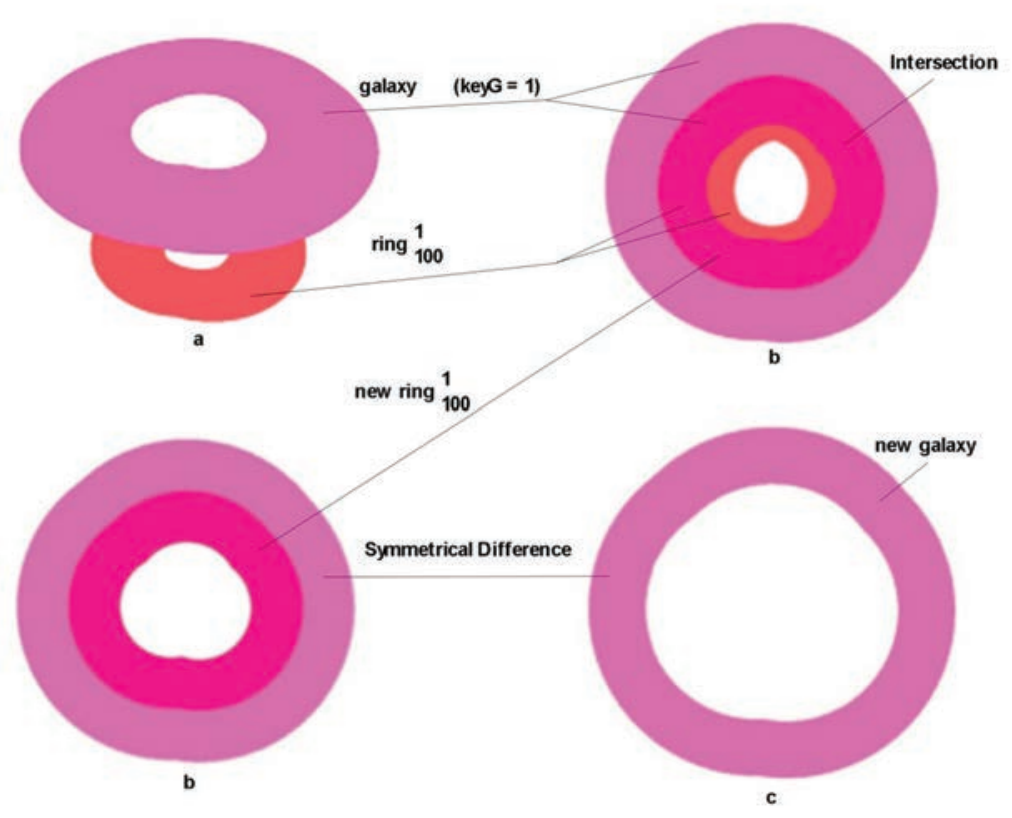

Figura 4 - Passos A2 a, b e c do algoritmo A

\section{2. Áreas de serviço de um subconjunto de features - algoritmo B}

Apresenta-se abaixo um outro algoritmo, B, para o mesmo efeito do anterior que, apesar de mais complexo, trata os conjuntos multiponto em simultâneo. No algoritmo B, galaxies é uma FC (variável) e MasterDiss uma FC em que cada feature é a fusão (ferramenta Dissolve) das SA das estruturas do estudo, associadas a um subconjunto e a um intervalo de impedância. Inicialmente, uma "galaxy" fusão (ferramenta Dissolve) de todas as SA correspondentes a um conjunto multiponto e a FC galaxies é a coleção dessas fusões. A FC (variável) rings corresponde à parte de MasterDiss relativa a um intervalo de impedância.

A ideia do algoritmo B é semelhante à do algoritmo A: cada galaxy é recortada intervalo a intervalo, removendo-se dela a SA correspondente a esse intervalo (de impedância). Só que isso é feito simultâneamente com todas as "galáxias" e rings (relativos a um intervalo de impedância). Temos pois um só ciclo, em vez de dois imbricados. 
Seja $I=\left\{I_{1}, \ldots, I_{k}\right\}$ o conjunto de intervalos de impedância, a cada qual corresponde um valor da chave keyQM.

B1. Retirar de MasterSA as features extrínsecas ao estudo.

B2. Usando a ferramenta Dissolve sobre keyQM e keyG, criar a FC MasterDiss a partir de MasterSA. (Figura 5)

B3. De MasterDiss criar, usando a ferramenta Dissolve sobre o campo keyG, a FC galaxies. (Figura 5)

B4. Na FC MasterDiss, dar um novo nome ao campo keyG, p.ex. $G N r$ (tal pode ser feito criando o novo campo, copiando para este os valores do anterior e eliminar keyB).

B5. $s a \leftarrow \emptyset$ (ferramentas Create Feature Class e/ou Delete Features). Na FC sa ficará o resultado deste algoritmo.

B6. Para $i=1, \ldots, k-1$ :

a. Por seleção, colocar os polígonos de masterDiss correspondentes a keyQM $M_{i}$ em rings.

b. rings $\leftarrow$ rings $\cap$ galaxies. [1]

c. Por seleção, deixar em rings apenas os poligonos tais que $G N r=k e y G$. Assim, em rings ficam as SA (dos vários conjuntos multiponto) correspondentes ao intervalo $I_{i}$. Eliminar de rings o campo keyG (útil mas não necessário). [2] (Figura 6c)

d. $S_{1} \leftarrow$ rings $\cup$ galaxies. [3] (Figura $6 \mathrm{~d}$ )

e. Por seleção, separar os polígonos de $S_{1} \operatorname{com} G N r=0$ (que são por sua vez colocados numa FC, $u_{0}$ ) dos com $G N r>0$ (que se colocam numa FC, $u_{1}$ ). [4] (Figura 6e)

f. Usando a ferramenta Delete Field, eliminar de $u_{1}$ o campo $G N r$. [5]

g. inside $\leftarrow u_{1} J_{\text {within }}^{s}$ rings (escolher a opção join_one_to_many, e desmarcar a opção Keep Target Features" - "junção interna"). [6] (Figura 6h)

h. Remover de $u_{1}$ os poligonos correspondentes a features de inside tais que keyG $=G N r$ e colocar os poligonos restantes numa FC, $u_{2}$. [7] (Figura $6 \mathrm{~h}$ )

i. Usando as ferramentas Append (ou Merge) adicionar à FC sa o conjunto de rings obtidos no passo B6.c. Simplificar o esquema de sa-o campo keyQM e um relativo à galáxia ( $G N r$ ou, preferivelmente, keyG) são obrigatórios.

j. Juntar as features $u_{0}$ e $u_{2}$ (ferramentas Merge ou Append), fundir (ferramenta Dissolve) segundo keyG e colocar o resultado em galaxies.

B7. No final da iteração $k-1$, na FC galaxies estarão os poligonos correspondentes ao último "ring", i.e. os poligonos respeitantes ao intervalo $I_{k}$. Esta FC deve ser apensa a sa (ferramentas Merge ou Append), mas de modo a que o campo GNr passe a chamar-se $k e y B$, e o campo keyQM seja preenchido na FC galaxies. (Figura 7)

B8. Fazer Dissolve (com a opção Multi_Part ativada) de sa sobre os campos relativos ao intervalo de impedância, keyQM, e à galáxia, keyG. A FC sa contém finalmente todas as áreas de serviço correspondentes a todos os conjuntos multiponto e intervalos de impedância. Para obter as SA de qualquer conjunto multiponto, basta usar o Query Builder para o selecionar. (Figura 8) 
Explicação do Algoritmo:

Ao longo do algoritmo deverá proceder-se à eliminação de eventuais campos espúrios e (não necessário) à transformação Multipart $\bigotimes$ Singlepart. São obrigatórios os campos keyQM (em sa e rings), GNr (em rings) e keyG (em galaxies e sa).

[0] Na figura 5 mostram-se em perspectiva galaxies e MasterDiss e respectivas tabelas, juntamente com os conjuntos multiponto. As Figs. 1, 2,5 e 6 ilustram o algoritmo, e a iteração $i=2$ do ciclo.

[1], [2] Relembrando a explicação da ferramenta Intersect vem que a figura 2 esclarece o passo B6.b. Quanto ao passo B6.c, as features para as quais $G N r=k e y G$ correspondem a divisões relativas à mesma galáxia (e conjunto multiponto) e portanto temos o equivalente a A2.b do algoritmo A, mas com as (três) intersecções obtidas em simultâneo. (Figura 6c incluindo a tabela de rings.)

[3] De notar que neste momento, as features de rings estão dentro das respectivas galáxias porque as partes que não as intersectavam foram-lhes retiradas.

[4] As divisões de $u_{O}$ não se sobrepõem a qualquer das features de rings, pelo que são agora parte da nova FC galaxies (a que será usada na próxima iteração). Quanto aos polígonos de $u_{1}$, alguns farão também parte, outros não. Os últimos devem ser eliminados.

[5] Este passo é recomendado, mas não obrigatório. Destina-se apenas a evitar o aparecimento de um campo, $G N r_{-} 1$, no próximo passo.

[6] e [7] É importante salientar que $u_{2}$ resulta de features de $u_{1} \mathrm{e}$ a seleção segundo $k e y G=G N r$ diz respeito a inside. Este passo pode ser executado de várias formas, vamos apresentar uma (Fig 6h):

1. Colocar uma chave candidata (keyy) e um campo "marcador" (remove) em $u_{1}$.

2. Colocar a zeros (ou false) todo a coluna relativa a remove.

3. Executar B6.g. Em inside vão ficar as divisões de $u_{1}$ que se encontram 
dentro de algum "ring"; em virtude da "completa" repartição produzida por Union, não existem em $u_{1}$ divisões que estejam parte dentro, parte fora de um ring e todas as divisões de rings estão replicadas em $u_{1}$.

4. Selecionar em as features tais que keyG $=G N r$ (ou seja, "provenientes" de galáxia e de anel relativos o mesmo conjunto multiponto); colocar o resultado em inside1.

5. Fazer a junção de atributo $u_{1} j_{\text {keyy }}^{a}$ inside1.

6. Se se optar por junção interna, colocar a 1 (ou true) o campo (exige editor activo); se se optar por junção externa, fazer o mesmo mas só nas linhas com correspondência (sem valores $N u l l$ ) nos campos da junção correspondentes a inside1.

7. Desfazer a junção e por seleção sobre $u_{1}$ colocar em $u_{2}$ as features de $u_{1}$ em que remove $=0$.

No contexto de um script estes sete (sub-)passos são muito mais fáceis de implementar (usando cursores), não sendo necessária a junção de atributo, mas antes um "varrimento" conjunto de $u_{1} \mathrm{e}$, ambos ordenados segundo keyy.

Imagine-se uma divisão que, independentemente do que se passa quanto a outras galáxias e rings, se sobreponha à galáxia 1 e aos rings 1 e 2 . Esta divisão aparece (pelo menos) duas vezes em $u_{1}$. Sejam 10 e 11 os valores de keyy nessas duas réplicas. Então nestas temos (keyG, $G N r, k e y y)=(1,1,10),(1,2,11)$. Eliminado o campo $G N r$ ficamos com $(k e y G, k e y y)=(1,10),(1,11)$. Ambas encontram réplicas iguais nos rings 1 e 2, pelo que após a junção espacial, essas duas réplicas vão originar em inside, quatro réplicas, a saber: $(\operatorname{keyG}, G N r, k e y y)=(1,1,10),(1,2,10)$, $(1,1,11),(1,2,11)$. Com a seleção $k e y G=G N r$ ficam somente duas: $(1,1,10)$ ,$(1,1,11)$. Repare-se que os dois valores de keyy, 10 e 11, aparecem, pelo que, após a junção de atributo ambas as réplicas existentes em $u_{1}$ vão ficar "marcadas" (remove $=1$ ) para eliminação. 
Com raciocínio similar, mas agora com $G N r \neq 1(k e y G \neq G N r)$ nos tripletos de $u_{1}$ acima, poderá concluír-se que as réplicas não ficam marcadas para eliminação, ou seja, são transferidas para $u_{2}$. Assim, todas as divisões de $u_{1}$ provenientes da galáxia 1 , que intersecta ring 1 são eliminadas, as que não intersectam ring 1 vão para $u_{2}$. $\operatorname{Em} u_{2}$ e em $u_{0}$ estão as divisões da "diferença simétrica” entre a galáxia 1 e o ring 1 .

Facilmente se verifica que o raciocínio acima é perfeitamente generalizável qualquer que seja o tipo de sobreposição, uma ou mais galáxias um ou mais rings. Aplicando o devido formalismo matemático, serve como demonstração.

Por último, convém notar que, tanto o algoritmo $\mathrm{A}$ como o $\mathrm{B}$, podem aplicar-se para o caso de MasterSA ser constituida não só por SA de pontos isolados mas também (e conjuntamente), ele próprio, por SA de conjuntos multiponto. Para tal basta considerar as SA de cada conjunto multiponto como as SA de um ponto isolado. Imagine-se que se tem já o resultado, , para um certo número de conjuntos multiponto, mas pretende-se acrescenter a um ou mais desses conjuntos mais alguns pontos. Em vez de usar-se novamente o NA (caso seja possível...) para os novos conjuntos multiponto (introduzidos um a um), pode aplicar-se qualquer dos algoritmos para actualizar. No caso da adição de novos pontos dizer respeito a somente um conjunto multiponto, o algoritmo A é claramente mais vantajoso, não só pela sua simplicidade como também porque, neste caso, o ciclo exterior é constituido por uma só iteração. De facto, ao resultado, , basta retirar as $\mathrm{SA}$ relativas a esse conjunto multiponto, usar-se o algoritmo para actualizar as SA desse conjunto e actualizar, pela adição das novas SA do conjunto multiponto (ferramentas Merge ou Append), em substituição das antigas. 


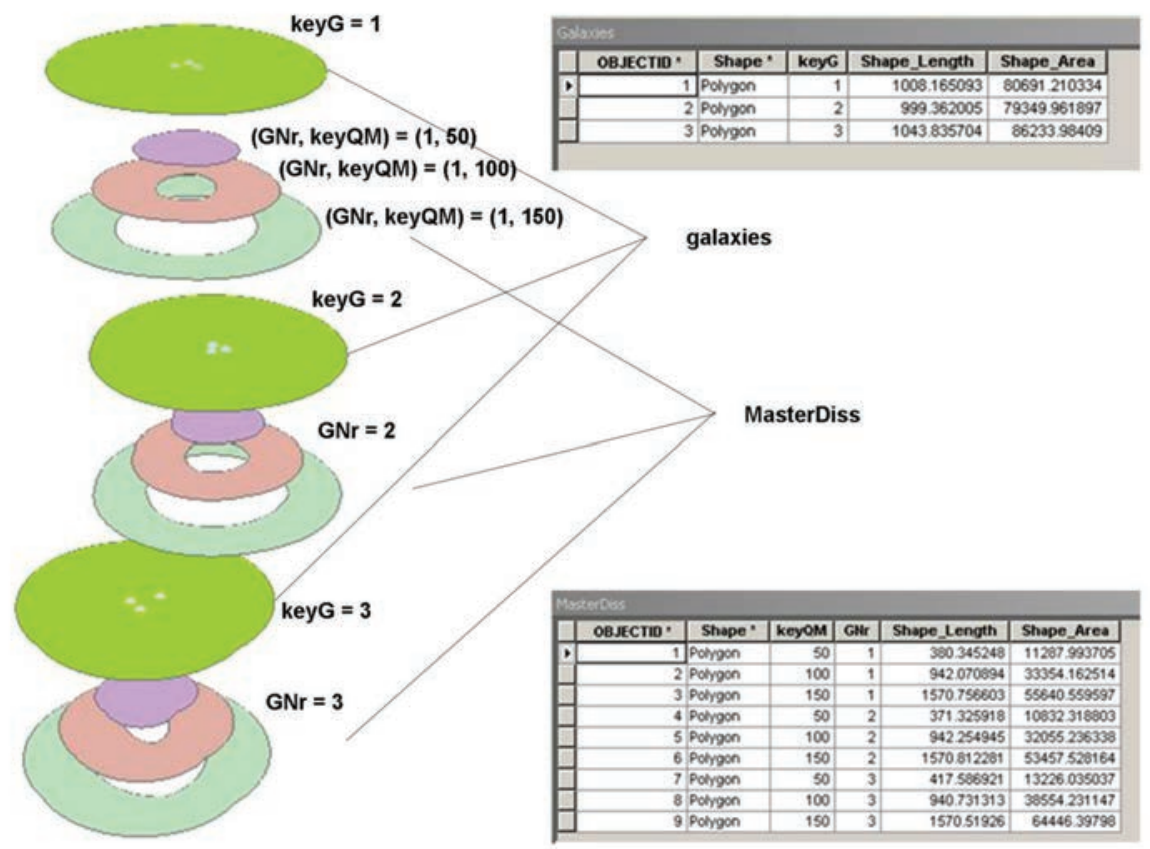

Figura 5 - FC galaxies e MasterDiss e tabelas associadas

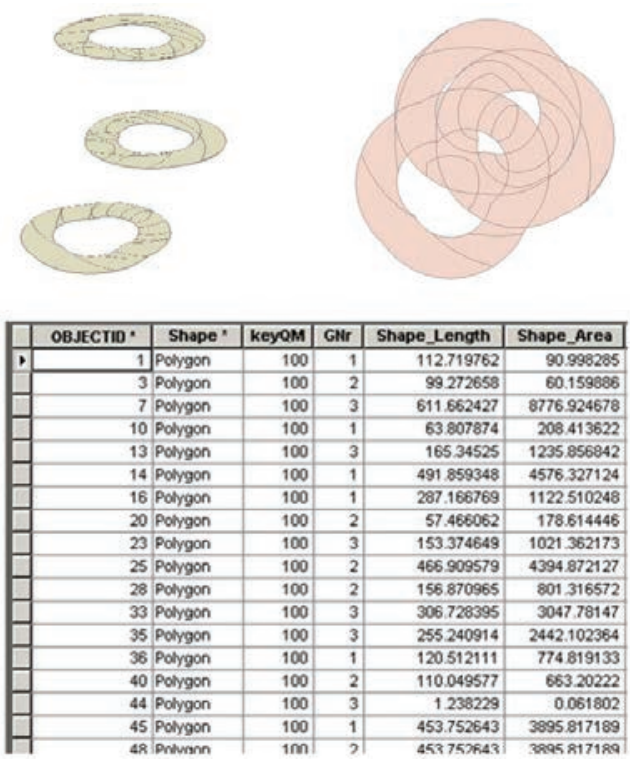

Figura 6c - FC rings e tabela associada, após B6.c 
Atas das I Jornadas Lusófonas de Ciências e Tecnologias de Informação Geográfica, Sessão 11, Artigo 48 Algumas técnicas SIG úteis à obtenção de áreas de serviço de conjuntos de pontos
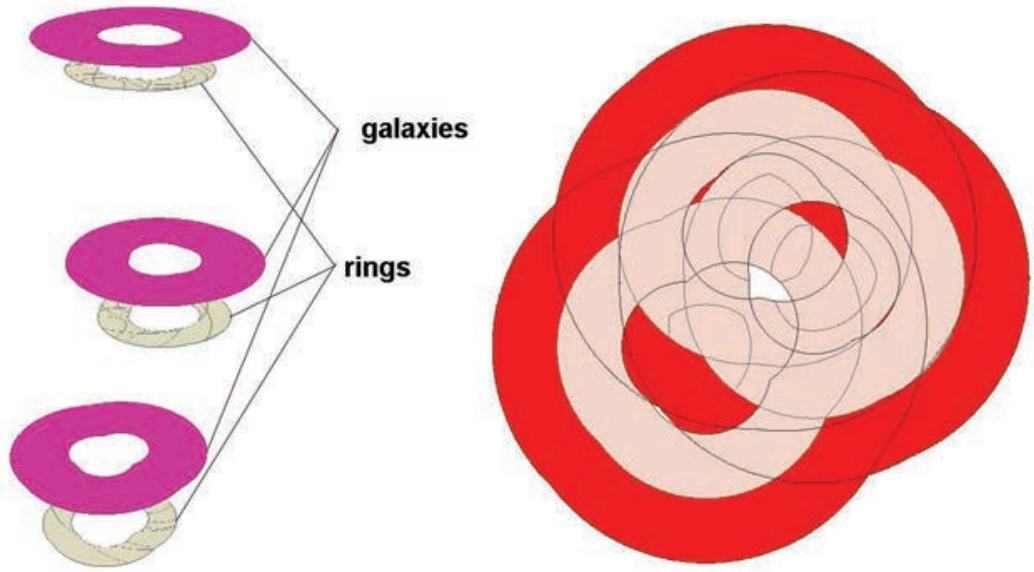

Figura 6d-galaxies, rings e $S_{1}$

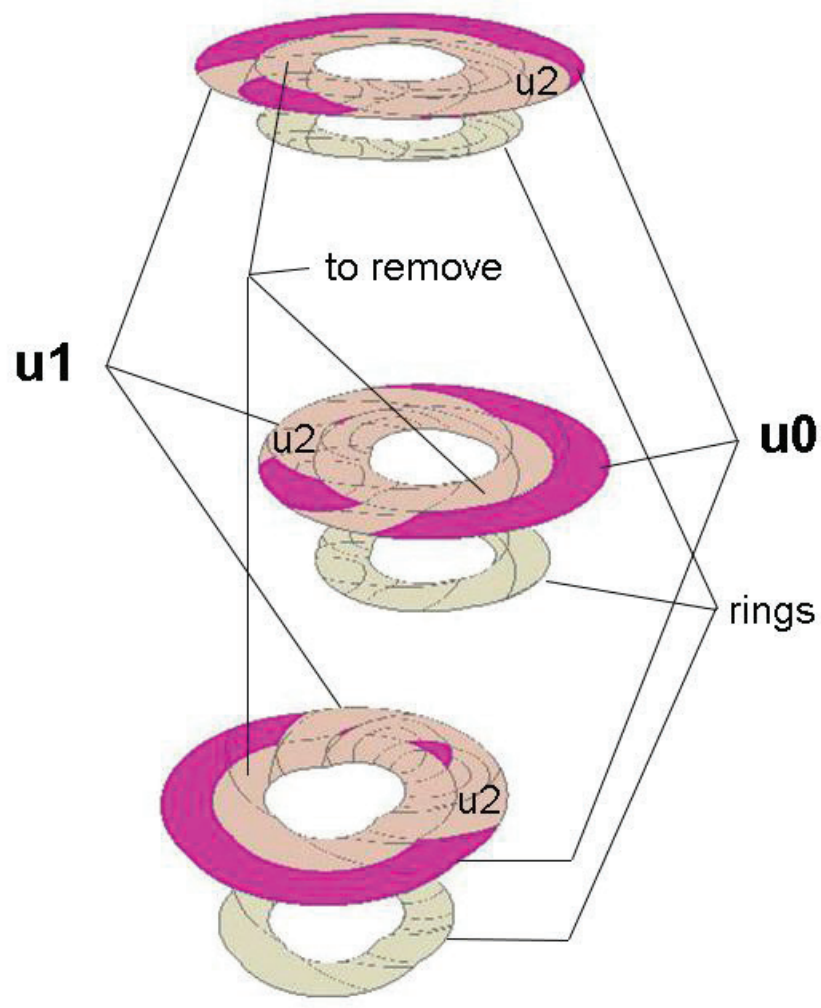

Figura $6 \mathrm{e}-u_{0}, u_{1}$ e rings 

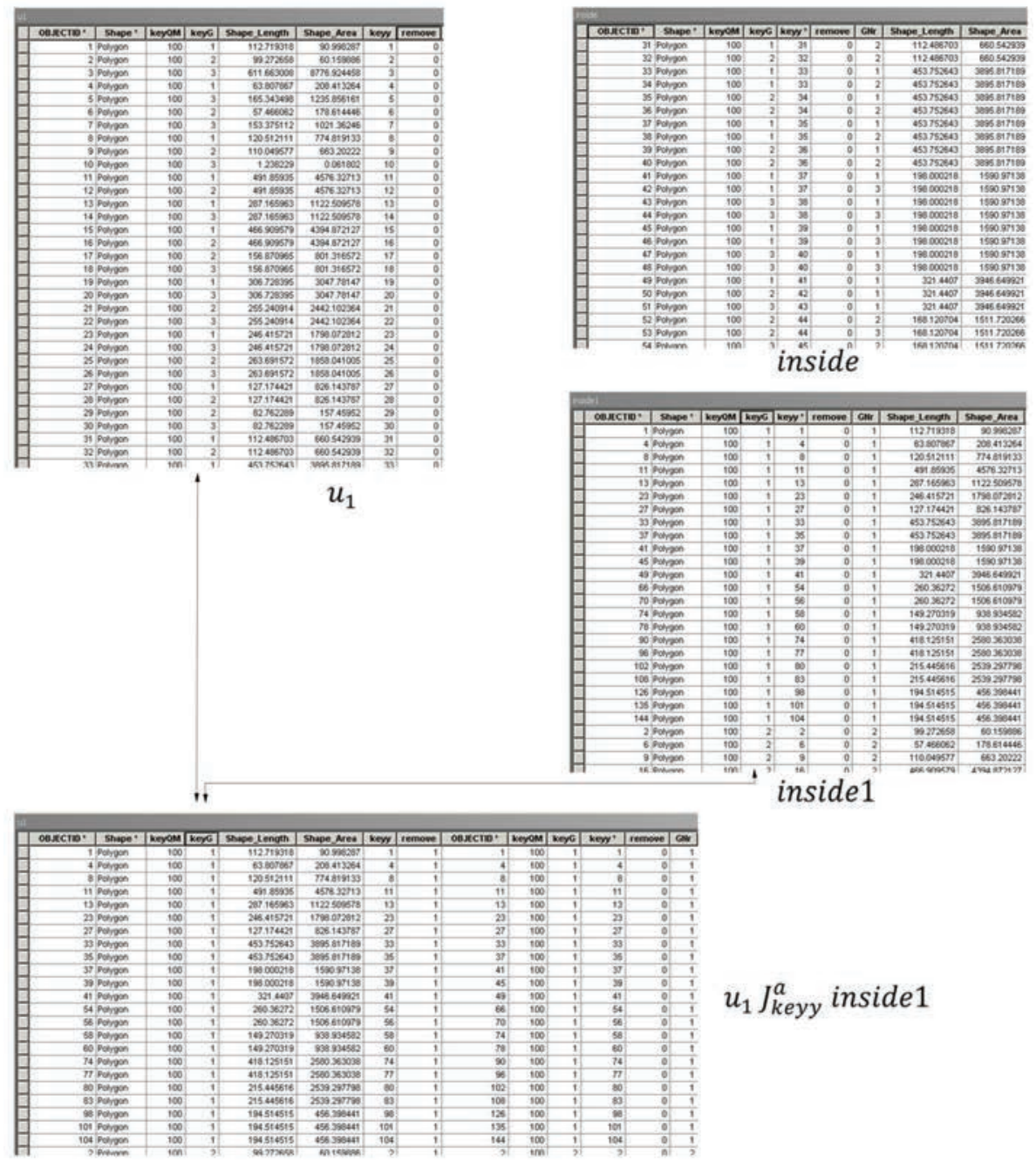

\section{$u_{1} J_{\text {keyy }}^{a}$ inside 1}

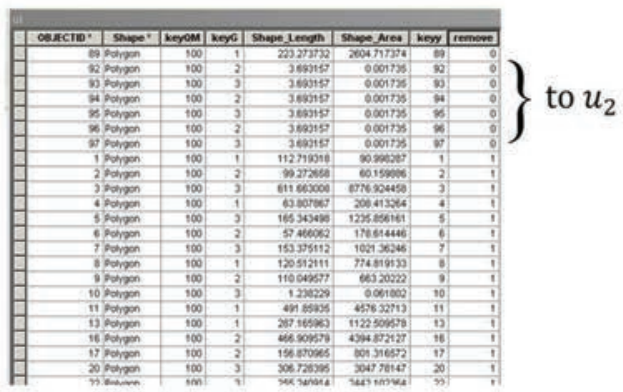

Figura 6h - Sequência de tabelas dos (sub-)passos de 6g e 6h (ver explicação) 


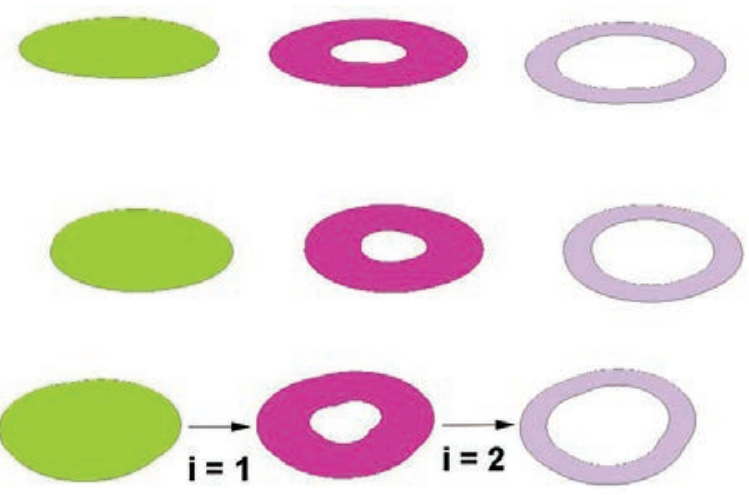

Figura 7 - Evolução da FC galaxies
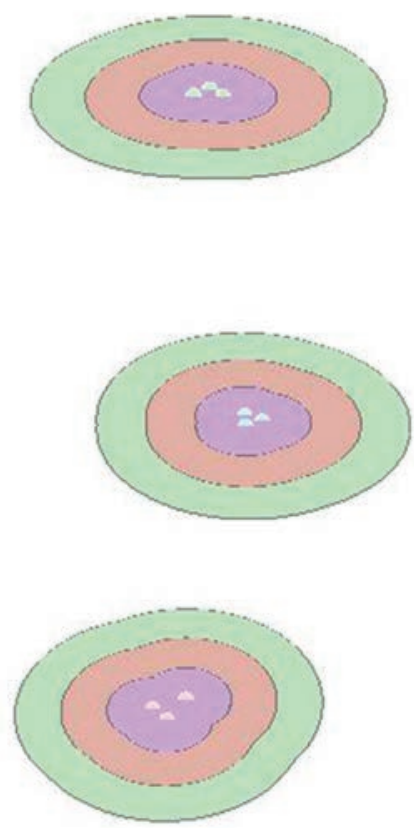

Figura 8 - FC $s a$ final 


\subsection{Um indicador de contorno}

Vamos agora supor que temos um conjunto de pontos ("procura" demand), por exemplo os centróides de uma mesh. Caso exista, a mesh é colocada na FC MeshPol. Supõe-se a procura na FC MeshPt. Ambas estas FC têm chave candidata $k e y B$.

Abaixo apresenta-se um algoritmo para determinar um indicador de contorno: o número de conjuntos multiponto que cada ponto de procura pode alcançar, até um dado valor máximo da impedância, imp. Convêm que imp seja extremo de um dos intervalos de impedância relativos às áreas de serviço. Porém, associando-se a cada área de serviço uma impedância, keyQM, o algoritmo também pode ser usado para o mesmo efeito, só que agora com erro dependente das amplitudes dos intervalos de impedância.

C1. Na tabela associada de MasterSA, criar e preencher um campo com os valores correspondentes de keyQM. Este passo não é estritamente necessário, mas facilita os restantes.

C2. Por seleção, ou através do Query Builder, retirar de MasterSA poligonos correspondentes a impedâncias acima de imp.

C3. Por junção espacial, criar a FC $S_{1}=M e s h P t J_{\text {within }}^{s}$ MasterSA, usando a opção one-to-many. $\mathrm{Em} S_{1}$ cada ponto de procura é então representado por uma pilha de pontos, um para cada poligono (SA) que o seu centróide (ou ponto de procura) intersecta.

C4. Usando a ferramenta Summary Statistics sobre $S_{1}$, criar uma tabela $T_{1}$, na qual para cada par $(k e y G, k e y B$ ) está presente o mínimo de keyQM (designe-se este campo porMin_keyQM).

C5. $S_{2} \leftarrow S_{1} J_{(\text {keyG }, \text { keyB })}^{a} T_{1}$.

C6. Remover de $\mathrm{S}_{2}$ todos os pontos para os quais keyQM $\neq$ Min_keyQM e colocar o resultado em $\mathrm{S}_{3}$.

C7. Colocar um campo chave candidata (key) em $S_{3}$.

C8. Usando Summary Statistics criar a tabela $T_{3}$ em que para cada valor do par (keyG, keyB) somente o primeiro valor de key aparece (no campo FIRST_key).

C9. Usando procedimento similar a $\mathrm{C5}$ e $\mathrm{C} 6$, agora usando key e FIRST_key criar a FC $S_{4}$ em que, para cada valor do par (keyG, keyB) somente a primeira feature de $S_{3}$ aparece. Ficamos pois em $S_{4}$, com a impedância de cada centróide da mesh ao ponto "mais próximo" de cada conjunto multiponto.

C10. Aplicando a ferramenta Summary Statistics (ou Frequency) sobre $S_{4}$, criar a tabela $T_{4}$, onde para cada keyB, o número de ocorrências de keyG é contado e colocado num campo. Cada um desses números corresponde ao número de subconjuntos multiponto à impedância imp ou menor do ponto keyB.

C11. O resultado final, sob a forma de uma FC de poligonos da mesh é obtido pela junção meshPol $J_{\text {keyB }}^{a} T_{4}$.

Algoritmo C 
Explicação do Algoritmo:

O tuplo (keyG, keyB) identifica um par (conjunto multiponto, ponto da mesh). C3 replica cada ponto da mesh (centróide ou ponto de procura) de forma a ficarem em cada "pilha" tantos pontos quantas as SA o centróide interseta. C4, C5 e C6 permitem determinar a menor impedância de cada centróide a cada conjunto multiponto, eliminando de $S_{2}$ os pontos de cada "pilha" a impedância superior a esse valor mínimo. Para prevenir o caso de um centróide estar à impedância mínima de mais do que um ponto de um conjunto multiponto, os passos C8 e C9 permitem escolher somente um dos pontos da pilha correspondente ao centróide, por sinal o primeiro, e eliminar os outros. Agora trata-se somente de contar quantos pontos restaram em cada "pilha" e obtêm-se o resultado final.

É possível usar uma parte de MasterSA em vez de toda essa FC, dependendo do que se pretende. Em particular, pode ser interessante considerar somente alguns subconjuntos de pontos. Note-se que se as áreas de serviço forem obtidas subconjunto a subconjunto, os passos $\mathrm{C} 4$ a C8 são desnecessários.

Uma variante curiosa deste algoritmo consiste em, num passo C6' selecionar de $S_{3}$ os valores de $k e y Q M_{i}$ correspondentes a um intervalo de impedância, ou ao intervalo união de vários intervalos adjacentes. Neste caso teríamos como resultado, e para cada célula da mesh o número de conjuntos multipontos situados nesse intervalo (união) de impedâncias.

\section{CASO DE ESTUdO}

Como aplicação dos algoritmos, apresenta-se um caso de estudo relativo à acessibilidade a algumas (14) das linhas dos Serviços Municipais de Transportes (rodoviários) Urbanos de Coimbra (SMTUC).

Aqui, $P$ é o conjunto das paragens das diversas linhas; a distância temporal pedonal a cada das paragens desempenha o papel de impedância. Como são estas os únicos pontos de acesso às linhas, torna-se claro que uma área de serviço (correspondente a um intervalo de tempo) 
relativa a uma linha é a área de serviço do conjunto multiponto constituído pelas suas paragens. Assim, neste caso keyG identifica uma linha e keyQM um intervalo de tempo. Como cada linha tem várias variantes, consideram-se, para cada linha as paragens de todas as suas variantes. Por aplicação do algoritmo $\mathrm{B}$ (ou $\mathrm{A}$ ), obteve- se SA relativas às diferentes linhas em estudo, destacando-se (Query Builder) o resultado para a linha 7, apresentado na figura 9. Nesta figura, os pontos de várias cores correspondem às paragens: uma cor por cada linha (as paragens da linha 7 encontram-se a branco). keyG é o código da linha. As zonas sombreadas a cores indicam áreas de serviço para acesso pedonal à paragem mais próxima da Linha 7 (a branco).

Pode parecer estranho considerarem-se valores tão elevados de impedância como 45min. Acontece, porém que a velocidade comercial média apresentada pelos SMTUC para os seus veículos é de aproximadamente 4 vezes o valor mais usual para a velocidade pedonal média. Assim, e para as SA relativas a impedâncias elevadas, o mapa dá também uma ideia do tempo de autocarro até à linha em causa, a qual é, notoriamente, de natureza circular.

$\mathrm{Na}$ figura 10 apresenta-se um resultado obtido pelo algoritmo C: o número de linhas de autocarros SMTUC a menos de 5 minutos a pé de cada quadrícula de uma mesh com quadrículas de 50m de lado. 


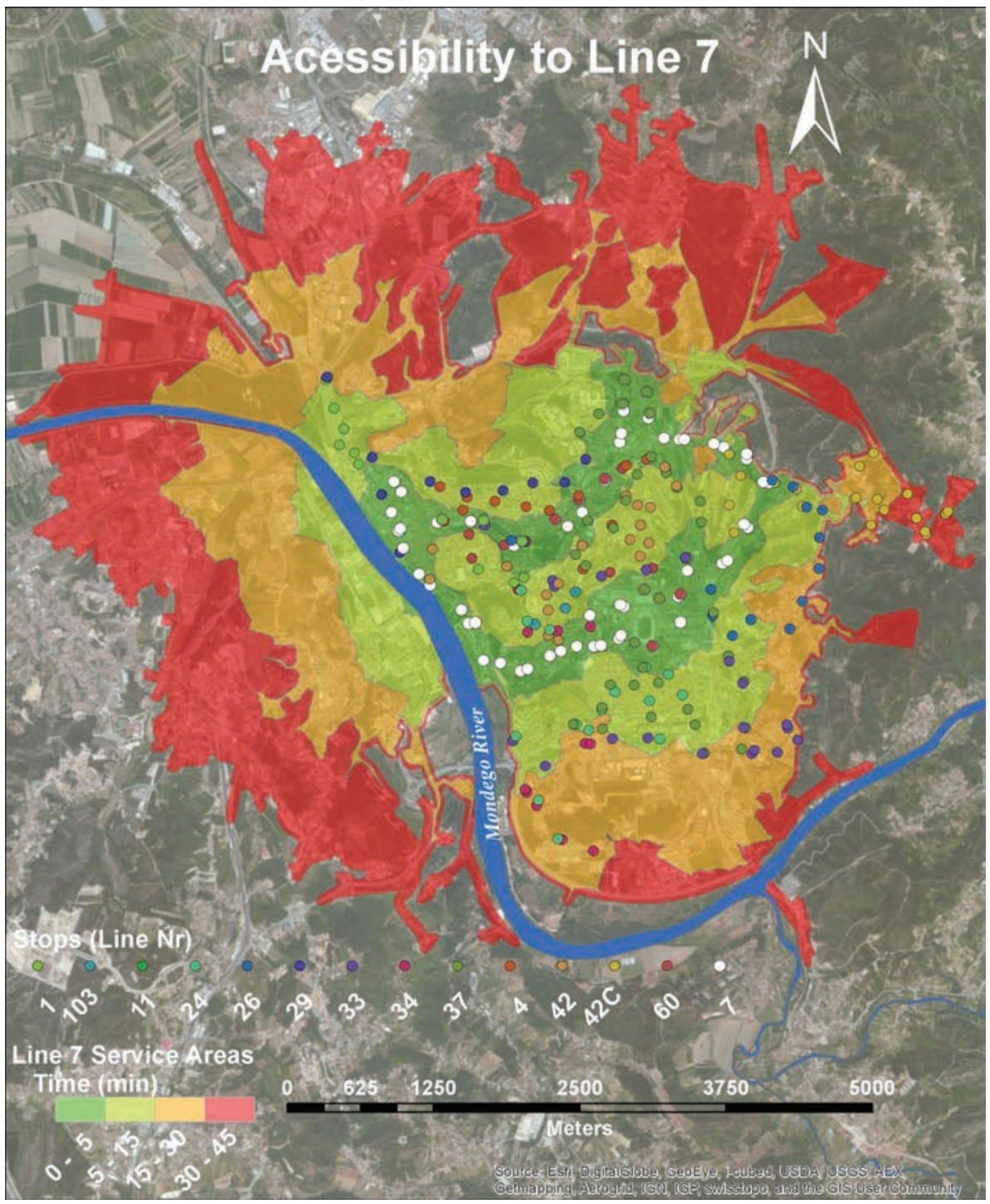

Figura 9 - Acessibilidade pedonal à Linha 7 dos SMTUC 


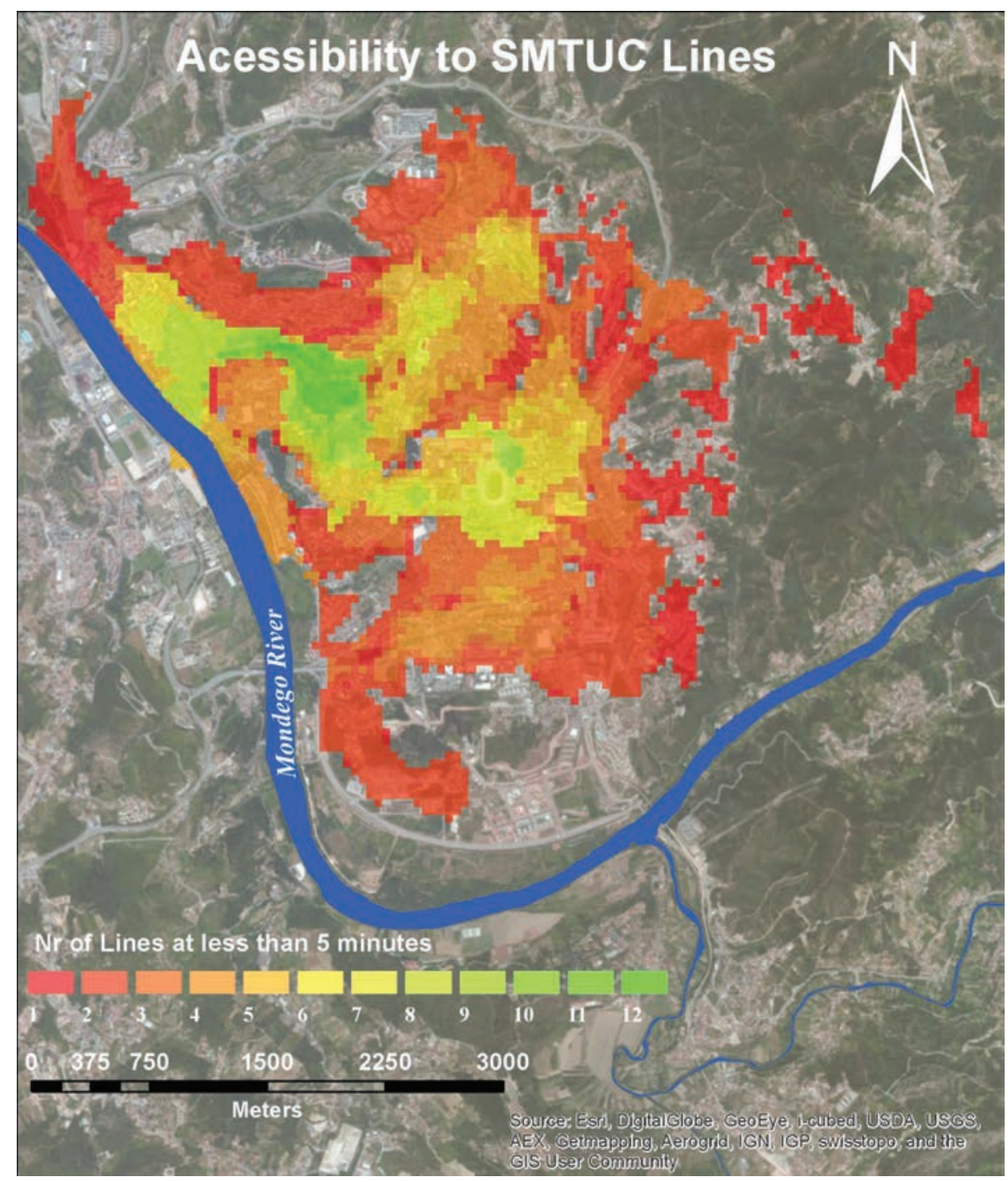

Figura 10 - Número de linhas de autocarros SMTUC a menos de 5 minutos a pé

\section{ConClusões}

Neste trabalho foi analisada a questão da acessibilidade a conjuntos de pontos. Apresentaram-se algumas técnicas SIG úteis à abordagem desse tema, baseadas na obtenção de áreas de serviço e manipulação de polígonos. Estas técnicas foram testadas no contexto de um caso de estudo. 
O caso de estudo debruçou-se sobre algumas linhas (14) de transportes públicos rodoviários da cidade de Coimbra, Portugal, mas a abordagem é completamente geral e extensível a outros tipos de equipamentos urbanos públicos, comerciais ou de serviços, vistos tanto isolada como agregadamente.

Os resultados permitem identificar deficiências e desequilíbrios oferta/ procura, fornecendo ao decisor elementos para o planeamento e gestão da cidade.

\section{AgRAdECIMENTOS}

Os autores agradecem à Fundação Portuguesa para a Ciência e a Tecnologia" (FCT), que financiou parcialmente este trabalho sob as referências "PEst-OE/ EEI/UI308/2014" e a iniciativa Energy for Sustainability da Universidade de Coimbra, suportada pelo projeto Energy and Mobility for Sustainable Regions (EMSURE) - CENTRO-07-0224-FEDER-002004.

\section{BIBLIOGRAFIA}

BEN-AKIVA, M. \& LERMAN, S.R. (1979) - "Disaggregate Travel and Mobility-Choice Models and Measures of Accessibility." in HENSHER, D.A. \& STOPHER, P.R. (eds.): Behavioural Travel Modeling, London: Croom-Helm, 654-679.

BOCAREJO, S. et al. (2012) - "Transport accessibility and social inequities: a tool for identification of mobility needs and evaluation of transport investments." Journal of Transport Geography, Vol. 24, 142-154.

BUTLER, J.A. (2008) - Designing Geodatabases for Transportation. ESRI Press, New York. 480 p.

DALVI, M.Q. \& MARTIN, K.M. (1976) - "The measurement of accessibility: some preliminary results." Transportation, Vol. 5, 17-42.

DE MONTIS, A. \& REGGIANI, A. (2012) - "JTG Special Section on Accessibility and Socio- Economic Activities: Methodological and Empirical Aspects." Guest editorial, Journal of Transport Geography, Vol. 25, 95-97.

ESRI (2007) - Working with ArcGIS network analyst. Environmental Systems Research Institute, EUA.

ESRI (2014) - ArcGIS 2008. Environmental Systems Research Institute, EUA. Disponível online no endereço url: http://resources.arcgis.com/content/ 
web-based-help (acedido em 30 de março 2014).

GEERTMAN, S. \& VAN ECK, J. (1995) - "GIS and models of accessibility potential: an application in planning." International Journal of Geographical Information Systems, Vol. 9, $\mathrm{N}^{\circ}$ 1, 67-80.

GEURS, K.T. \& VAN ECK, J.T.R. (2001) - Accessibility Measures: review and applications. RIVM Report 408505/006. Utrecht University, Países Baixos.

GEURS, K.T. \& VAN WEE, B. (2004) - "Accessibility evaluation of land-use and transport strategies: review and research directions.” Journal of Transport Geography, Vol. 12, No 2, 127-140.

HALDEN, D. et al. (2005) - "Accessibility analysis literature review." Working paper. Disponível online no endereço url: (acedido em 30 de março 2014) http:// home.wmin.ac.uk/transport/download/SAMP_WP3_Accessibility_Modelling. pdf

HANSEN, W. (1959) - "How Accessibility Shapes Land Use." Journal of the American Institute of Planners, Vol. 25, 73-76.

HEYWOOD, I. et al. (2011) - An Introduction to Geographical Information Systems, $4^{\mathrm{a}}$ Ed. Pearson Education Limited Ed., Harlow, Reino Unido.

IACONO, M. et al (2010) - "Measuring non-motorized accessibility: issues, alternatives, and execution.” Journal of Transport Geography, Vol. 18, $\mathrm{N}^{\mathrm{o}}$ 1, 133-140.

LITMAN, T. (2003) - Accessibility: Defining, Evaluating and Improving Accessibility. Victoria Transport Policy Institute. Disponível online no endereço url: www.vtpi.org (acedido em 30 de março 2014).

LONGLEY, P.A. et al. (2011) - Geographic Information Systems \& Science. Wiley, Hoboken (NJ), EUA.

MAVOA, S. et al. (2012) - "GIS based destination accessibility via public transit and walking in Auckland, New Zealand.” Journal of Transport Geography, Vol. 20, 15- 22.

PÁEZ, A. et al. (2012) - "Measuring accessibility: positive and normative implementations of various accessibility indicators." Journal of Transport Geography, Vol. 25, 141- 153.

POOLER, J.A. (1995) - "The use of spatial separation in the measurement of transport accessibility." Transportation Research A, Vol. 29, No 6, 421-427 
Série Documentos

Imprensa da Universidade de Coimbra

Coimbra University Press

2015

- U M

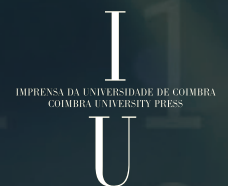

